\title{
Tempo de atuação do profissional enfermeiro - Minas Gerais
}

Recebido em: 26/07/2011

Aceito em: 23/11/2011
Lenice de Castro Mendes Villela ${ }^{1}$ Elizabeth Perez Galastro'

Maria Édila Abreu Freitas ${ }^{2}$ Mariana Silva Guimarães Santos ${ }^{3}$ Karine Antunes Marques Notaro ${ }^{4}$

Estudo tipo série histórica acerca do tempo médio de atuação dos enfermeiros que por algum motivo cancelaram sua inscrição no Conselho Regional de Enfermagem de Minas Gerais (COREN-MG) entre 1995 e 2006. As variáveis incluem tempo e local de atuação profissional, sexo, faixa etária e motivo do cancelamento. $O$ cancelamento das inscrições foi de 1.035 enfermeiros: destes, $92,2 \%$ do sexo feminino. $O$ tempo de atuação do enfermeiro no mercado de trabalho aproxima-se de 50\% nos dez primeiros anos e $25 \%$ entre 11 a 20 anos. No primeiro ano de atuação, há uma evasão de $10 \%$.

Descritores: Exercício Profissional, Recursos Humanos de Enfermagem, Satisfação no Emprego.

\section{Time performance of the professional nurse - Minas Gerais}

A time series study on average time of nurse practice before cancelling their Regional Council of Nursing of Minas Gerais (MG-COREN) was conducted using data from 1995 to 2006. Variables included time and location of professional activity, gender, age and reason for cancellation. Cancellation of entries was 1,035 nurses of which $92.2 \%$ were female. The operating time of the nurse in the labor market was approaching $50 \%$ in the first ten years and $25 \%$ from 11 to 20 years. In the first year of work, there was a $10 \%$ drop out.

Descriptors: Professional Practice, Nursing Staff, Job Satisfaction.

\section{Tiempo de actuación del professional enfermeiro - Minas Gerais}

Estudio del tipo serie histórica relacionado al tiempo medio de actuación de los enfermeros que por algún motivo cancelaron su inscripción en el Consejo Regional de Enfermería, Minas Gerais (COREN-MG), entre 1995 y 2006. Las variables incluyen tiempo y local de actuación profesional, sexo, grupo etario y motivo de la cancelación. La cancelación de las inscripciones fue de 1.035 enfermeros, siendo $92.2 \%$ del sexo femenino. El tiempo de actuación del enfermero se aproxima de 50\% en los diez primeros años y 25\% entre 11 a 20 años. En el primer año de actuación hay una evasión de $10 \%$. Descriptores: Práctica Profesional, Personal de Enfermería, Satisfacción en el Trabajo.

\section{INTRODUÇÃO}

$\mathrm{N}$ o Brasil, o mercado de trabalho em saúde apresentou transições nas décadas de 1970 e 1980, com uma economia voltada para a industrialização, o que resultou para o setor saúde na expansão das instituições hospitalares, com um modelo hospitalocêntrico. Segundo Laurtert ${ }^{(1)}$, esse modelo determinou uma assistência de baixo custo, desqualificada, uma vez que o propósito era o de reduzir os gastos e insumos e empregar recursos humanos não qualificados e em menor quantidade. Tal expansão não foi observada na melhoria das condições de trabalho, pelo contrário, expandiu-se a precarização do trabalho na área da saúde, especificamente para os profissionais da enfermagem. Neste contexto, o Estado enquanto precursor do capitalismo exerceu sua influência sobre o processo de trabalho da enfermagem brasileira, cujo fazer se pautava por uma estrutura econômica, política, social e ideológica do país (2-3).

Na década de 1990, as mudanças no setor de saúde aconteceram com a Reforma Sanitária e com a implantação do Sistema Único de Saúde (SUS). A partir dessa época surge um novo modelo de organização fundamentado no processo saúde/ doença e os enfermeiros passam a repensar a prática clínica com suas limitações, seu caráter medicalizante e o seu papel incipiente no controle social. Nesse processo, os enfermeiros começam a perceber o indivíduo como objeto de sua ação, isto é, pessoas que vivenciam determinados sofrimentos, agravos e necessitam de um atendimento integral, o que contribuiu para mudanças mais substantivas nas práticas assistenciais ${ }^{(4)}$. Nesse modelo assistencial, a enfermagem destaca-se como prática social inserida no sistema de saúde universal, igualitário, integral, de qualidade e fundamentado nos princípios de cidadania em direção a uma nova fase de consolidação da sua força de trabalho, especialmente no âmbito da saúde coletiva.

Esse enfoque constitui uma nova experiência de atuação dos profissionais enfermeiros no contexto das famílias e consequentemente exigem outros conhecimentos específicos com uma abordagem voltada para o coletivo, fora do contexto tradicional de atendimento ao cliente individual. A complexidade dessa estrutura reorienta os profissionais de saúde para um novo processo de trabalho e modos de inserção no cenário coletivo( ${ }^{(5)}$.

Assim, o enfermeiro amplia seu conhecimento científico e sua autonomia nas atividades assistenciais e coletivas, tanto na rede hospitalar como na assistência primária de saúde. No entanto, estudos têm demonstrado uma evasão dos enfermeiros

1 Enfermeira. Doutora em Enfermagem. Professor Adjunto UFMG. E-mail: lenicevillela@gmail.com.

2 Enfermeira. Doutora em Enfermagem. Professora Associada da UFMG.

3 Enfermeira. Especialista em Enfermagem Geriátrica. Atua no Hospital Mater Dei - Belo Horizonte/MG.

4 Enfermeira. Especialista em Enfermagem Hospitalar. Atua no Hospital Mater Dei - Belo Horizonte/MG. 
frente ao exercício profissional devido a problemas relacionados principalmente a longas e variadas jornadas de trabalho, baixos salários, indefinição de sua prática, funções e outras questões de cunho social(6-7). De acordo com Angerami ${ }^{(8)}$, o abandono do mercado de trabalho corresponde a um percentual de $11,4 \%$, o que pode ser de ordem familiar e profissional, baixos salários e perda da motivação, razões também citadas pelos profissionais que nunca atuaram na profissão. No que se refere aos anos trabalhados pelo enfermeiro no Estado de Minas Gerais, segundo Mendes ${ }^{(9)}$, até a década de 1980 a vida média do trabalho do enfermeiro girava em torno de dez anos.

Entretanto, a partir da década de 1990, o enfermeiro vem enfrentando uma nova transição decorrente do aumento crescente do número de escolas de graduação de enfermagem, e, consequentemente, de uma possível saturação do mercado de trabalho.

Frente a essas considerações e às escassas pesquisas referentes às condições de trabalho do enfermeiro, a permanência e abandono de sua profissão e o impacto decorrente do maior número de profissionais disponibilizados para o mercado de trabalho, justifica-se tal estudo.

Este artigo pretende, portanto, delinear o tempo de atuação e os motivos de cancelamento e abandono das atividades do profissional enfermeiro no Conselho Regional de Enfermagem de Minas Gerais (COREN-MG) no período de 1995 a 2006.

\section{METODOLOGIA}

O presente estudo é do tipo série histórica, retrospectivo transversal, acerca do tempo médio de atuação dos profissionais enfermeiros, que por algum motivo cancelaram sua inscrição no Conselho Regional de Enfermagem de Minas Gerais (CORENMG), no período de 1995 a 2006. O desenvolvimento de todas as etapas do estudo ocorreu na sede do COREN-MG, situado à Rua da Bahia, $n^{\circ}$ 916, Centro, Belo Horizonte - MG. A coleta de dados foi realizada em dois momentos, no período de 1995 a 2000 e entre 2001 e 2006. No primeiro período, utilizou-se o livro de registros de cancelamento das atividades profissionais dos inscritos, onde realizou-se a busca das informações referentes ao tempo e local de atuação do profissional enfermeiro, motivo do cancelamento, faixa etária e sexo. No segundo período, de 2001 a 2006, o livro de registros foi substituído por um banco de dados informatizado, quando da implantação do sistema INCORP/COREN-MG, que disponibilizou as informações referidas anteriormente. As variáveis estudadas incluem tempo e local de atuação profissional, sexo, faixa etária e motivo do cancelamento. Para esta última variável destaca-se a migração, ou seja, a transferência entre os estados e países, aposentadoria, falecimento, abandono da profissão e inscrição remida. A variável "outros" inclui as solicitações dos enfermeiros que não informaram o motivo do cancelamento do exercício profissional. Para consolidação e análise dos dados, foram agrupados os períodos pesquisados, utilizando-se planilhas construídas no software Excell.

Atendendo à Resolução 196/96 do Ministério da Saúde, a pesquisa foi autorizada pelo Conselho Regional de Enfermagem de Minas Gerais e submetida ao Comitê de Ética em Pesquisa
Figura 1 - Motivos do cancelamento dos inscritos no CORENMG, 1995 a 2006.

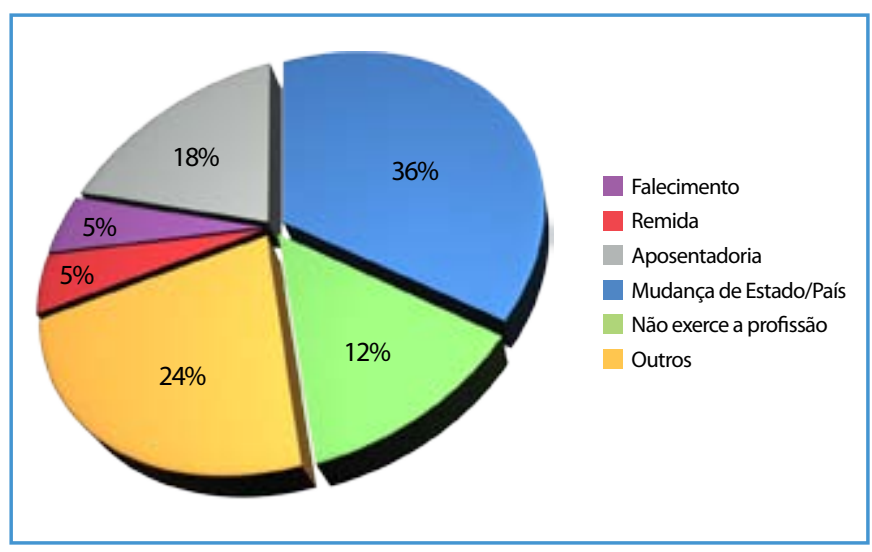

Fonte: Livro de Registro e INCORP/COREN-MG

da Universidade Federal de Minas Gerais (COEP/UFMG), sendo aprovada sob o parecer n ETIC 0390.0.203.000 - 10.

\section{RESULTADOS E DISCUSSÃO}

Os resultados indicam que no período entre 1995 a 2006, o cancelamento das inscrições no COREN-MG foi solicitado por 1.035 enfermeiros. Destes, $92,2 \%$ do sexo feminino, o que traduz as características comuns da identidade profissional da enfermagem. Alguns estudos descrevem a prevalência das mulheres no exercício profissional, que pode ser justificada pela predominância da força de trabalho feminino nas atividades referentes ao cuidado com as pessoas ${ }^{(7-10)}$.

Na figura 1, observa-se que o principal motivo de cancelamento dos inscritos refere-se à mudança dos enfermeiros para outros Estados ou países, por motivos não explicitados, aposentadorias ou por não exercerem a profissão. A migração dos enfermeiros do Estado de Minas Gerais pode justificar-se pela localização geográfica das instituições de ensino formadoras desses profissionais, que fazem limite com outros Estados e que os absorvem por proximidade, por melhores salários, oportunidades de trabalho e para capacitações, seja lato sensu ou stricto sensu.

A variável "outros" (24\%), apesar de se destacar, dificulta a

Figura 2 - Intervalo de tempo de atuação do enfermeiro inscritos no COREN-MG, 1995 a 2006.

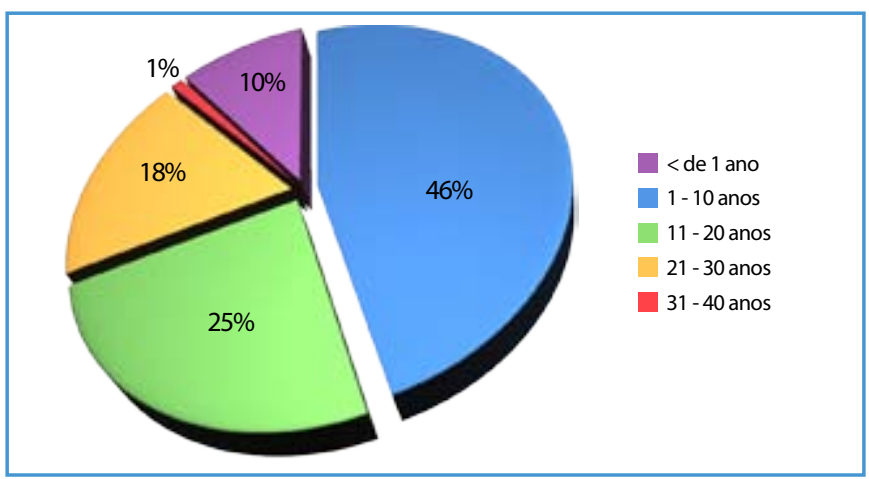

Fonte: Livro de Registro e INCORP/COREN-MG 
compreensão dos motivos, podendo constituir uma limitação dos registros ou subnotificação dos dados, entre diversos problemas do sistema de informação, considerando que, para o estudo, o COREN disponibilizava naquela época dois bancos de dados. Outra variável importante refere-se ao não exercício da profissão (12\%), que se justifica pelo papel que a mulher desempenha na sociedade. Nesse sentido, a mulher enfrenta as condições oferecidas pelo mercado de trabalho e sua potencialidade neste espaço, convivendo com a dinâmica do serviço que lhe é atribuído e, ao mesmo tempo, com o gerenciamento pessoal. Assim, as condições de trabalho referentes ao desgaste físico e emocional do enfermeiro, baixos salários, insatisfação profissional, falta de motivação, desprestígio social e os cuidados com a família são fatores que ainda interferem na decisão de se inserir ou não no mercado de trabalho ou optar por outras profissões na área da saúde ${ }^{(6,11-13)}$. O cancelamento precoce das atividades profissionais resulta em perdas financeiras para a formação do profissional, seja no ensino público, onde o Estado arca com os gastos nesta formação, seja nas escolas privadas, quando há um investimento pessoal ou familiar. Em ambas as situações, vale a reflexão sobre o investimento perdido na formação de indivíduos em idade produtiva que não restituem à sociedade este trabalho ${ }^{(7)}$.

Em relação ao período de atuação do profissional verifica-se na figura 2 que o tempo de participação do enfermeiro no mercado de trabalho aproxima-se de $50 \%$ nos dez primeiros anos do exercício profissional e $25 \%$ entre 11 a 20 anos. Ressalta-se que no primeiro ano de atuação profissional há uma evasão $10 \%$ do enfermeiro do mercado de trabalho. Diante desses resultados ocorre um esvaziamento crescente gradativo de aproximadamente $89 \%$ da força de trabalho, o que representa perda dos anos potenciais de estudo, custo social para a graduação e até mesmo de investimentos pessoais.
Para Taylor ${ }^{(14)}$, a atuação do profissional enfermeiro, a partir da sua graduação, apresenta um decréscimo de $60 \%$ no total da força de trabalho nos dez primeiros anos de seu exercício. Outros estudos demonstram que o período de serviço do enfermeiro, a partir de seu ingresso, está reduzido a cerca de dez anos $s^{(9,13,15)}$. Aliado a isso há uma perda social e econômica, uma vez que a maturidade profissional é essencial para a compreensão do processo de trabalho e da melhoria da saúde da população.

\section{CONSIDERAÇÕES FINAIS}

O estudo permitiu constatar que o tempo de atuação do profissional e os motivos do abandono da profissão do enfermeiro no Estado de Minas Gerais, no período de 1995 a 2006, são semelhantes a estudos apresentados em várias instâncias, seja no âmbito nacional e/ou internacional.

Os fatores determinantes para a evasão do profissional estão vinculados aos processos históricos, sociais, econômicos, culturais e científicos. A questão de gênero complementa esses fatores, visto que há um contingente de profissionais do sexo feminino que exerce a arte de cuidar e traz para o mundo público as mesmas características do mundo privado, ou seja, a casa, a família, os filhos. Há um conjunto de circunstâncias, desde o estritamente pessoal, perpassando pela sua formação profissional, estrutura social e a organização do próprio sistema de saúde, incluindo os aspectos políticos.

$\mathrm{O}$ conhecimento e a investigação desses determinantes contribuirão para o delineamento das dificuldades enfrentadas, que possivelmente possam interferir no tempo médio do trabalho do enfermeiro. A partir desta investigação, novas pesquisas poderão ser desenvolvidas para elucidar e proporcionar novos conhecimentos sobre a problemática da inserção do profissional na sociedade, da sua formação, além de investigar a necessidade de recursos humanos qualificados e a complexidade dos serviços de saúde.

\section{Referências}

1. Lautert L. O desgaste profissional do enfermeiro [tese]. Salamanca (ES):

Universidade Pontifícia Salamanca; 1995.

2. Pires D. Hegemonia médica na saúde e a enfermagem - Brasil 1500 a 1930. São

Paulo: Cortez; 1989

3. Pires D, Gelbcke FL, Matos E. Organização do trabalho em enfermagem:

implicaçōes no fazer e viver dos trabalhadores de nível médio. Trab Educ Saúde.

2004;2(2):311-20.

4. Carvalho SR, Cunha GT. A gestão da atenção na saúde: elementos para se

pensar a mudança da organização na saúde. In: Campos GWS. Tratado de saúde

coletiva. São Paulo: Hucitec, Rio de Janeiro: Fiocruz; 2006.

5. Cianciarullo TI. A questão do desenvolvimento do conhecimento na saúde:

o PSF no cenário atual. In: Cianciarullo TI, Silva GTR, Cunha ICKO. Uma nova

estratégia em foco: o Programa de Saúde da Família - identificando as suas

características no cenário do SUS. São Paulo: Ícone; 2005.

6. Duarte GG, Angerami ELS, Gomes DLS, Mendes IJM. Vida média de labor dos

enfermeiros egressos da Escola de Enfermagem de Ribeirão Preto, USP - Brasil.

Rev Latinoam Enferm. 2000;8(6):91-5

7. Secaf V, Rodrigues ARF. Enfermeiros que deixaram de exercer a enfermagem: por que? Rev Latinoam Enferm. 1998;6(2):5-11.

8. Angerami ELS, Gomes DLS, Mendes IJM. Estudo da permanência dos enfermeiros no trabalho. Rev Latinoam Enferm. 2000;8(6):91-5.

9. Mendes DC. Recursos humanos em enfermagem. Adequação da formação a utilização. In: Congresso Brasileiro de Enfermagem, 34. Porto Alegre, 1982. Anais: Porto Alegre (PA): Aben; 1982. p. 78-84.

10. Martins C, Kobayashi RM, Ayoub AC, Leite MMJ. Perfil do enfermeiro e

necessidades de desenvolvimento de competência profissional. Texto Contexto Enferm. 2006;15(3):472-8.

11. Marziale MHP. Enfermeiros apontam as inadequadas condiçōes de trabalho como responsáveis pela deterioração da qualidade da assistência de enfermagem. Rev Latinoam Enferm. 2001;9(3):1-5.

12. Associação Brasileira de Enfermagem. Levantamento de recursos e necessidades em enfermagem no Brasil - 1956/1958. Brasília: ABEN; 1980. 13. Estryn-Behar M, Poinsignon M. Travailler à l'hôpital. Paris: Berger-Levrault; 1989. 14. Taylor, C.E., Dirican, R., Deuschle, K.W. Helath manpower planning in Turkey: an international reseach case study. Baltimore: Johns Hopkins Press; 1976. 15. Caviochioli A. Profissionalitá infermieristica: element essenziali, strumenti operative. In: Anselmi ML. II quadro de riferimento per la formulaziena del budget del personale infermieristico nelle aziende sanitarie. Milano: University of Birgman/Universitá Commerciale "L. Bocconi" Scuola direzione aziendale; 1996-1997. 